72

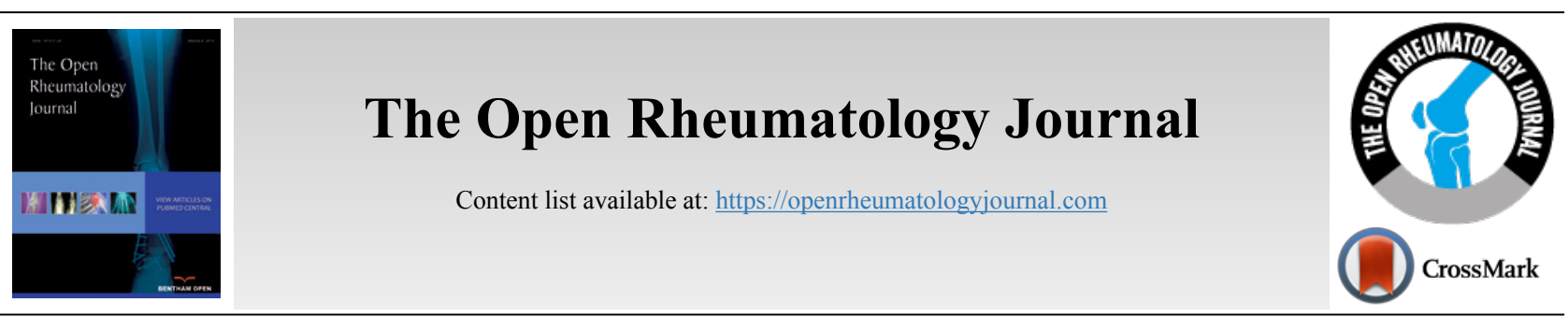

REVIEW ARTICLE

\title{
Regulatory Perspectives on Biopharmaceuticals for Chronic Inflammatory Diseases in North Africa: A Narrative Review
}

\author{
Ouafa Mkinsi ${ }^{1}$, Salima Lefkir-Tafiani ${ }^{2}$, Héla S, Srairi ${ }^{3}$, Samir Kochbati ${ }^{4}$, Yasser M. El Dershaby ${ }^{5}$, Mohamed M. El \\ Azhari ${ }^{5}$, Sabrina A-I. Midjek ${ }^{6}$ and Aicha Ladjouze-Rezig ${ }^{7 *}$
}

'Service de Rhumatologie, CHU Ibn Rochd, Casablanca, 20250, Morocco

${ }^{2}$ Service de Rhumatologie, CHU Béni Messous, Algiers, 16206, Algeria

${ }^{3}$ Department of Rheumatology, La Rabta Hospital, Tunis, 1007, Tunisia

${ }^{4}$ Department of Rheumatology, Habib Thameur Hospital, Tunis, 1069, Tunisia

${ }^{5}$ Pfizer, Dubai, 29553, United Arab Emirates

${ }^{6}$ Pfizer, Algiers, 16035, Algeria

Department of Rheumatology, University of Algiers 1, 16306, Algiers, Algeria

\begin{abstract}
Introduction of innovative biopharmaceuticals has dramatically changed the treatment of chronic inflammatory diseases, but access to these very effective agents may be limited by economic constraints in some regions. The development of biosimilar products at a lower cost may allow wider access to treatment, but rigorous scientific evaluation is required to ensure similar quality, efficacy, and safety. The World Health Organization, European Medicines Agency, and United States Food and Drug Administration have created stringent guidelines for biosimilar regulatory approval, stipulating that high similarity be demonstrated in comprehensive comparability studies. Although these regulatory standards have been adapted in many countries, the legal/regulatory frameworks required for biosimilar authorization remain in development elsewhere, including North Africa. In some countries, "intended copies" are available despite inadequate evidence of comparability to the reference product and failure to satisfy biosimilar regulatory requirements. In North Africa, as the regulatory pathway for biosimilars is established, regulators will address several important challenges, including criteria for comparability, switching/substitution, post-marketing monitoring/risk management, and product naming conventions. Caution is advised to ensure that lower cost and broader access are not achieved at the expense of patient safety, and educational initiatives should be undertaken for clinicians/patients. In this review, we define the various types of biopharmaceuticals currently available for the treatment of chronic inflammatory disease, provide an overview of regulatory requirements for biosimilar approval and an update on the availability of these agents globally and in North Africa, and discuss crucial concerns related to their use from the viewpoint of North African rheumatologists.
\end{abstract}

Keywords:: Rheumatic diseases, Biopharmaceutical, Biosimilar, Intended copy, Switching, Chronic inflammatory.

\begin{tabular}{|l|l|l|r}
\hline Article History & Received: February 07, 2019 & Revised: June 14, 2019 & Accepted: July 12, 2019
\end{tabular}

\section{INTRODUCTION}

By the end of the $20^{\text {th }}$ century, advances in science and technology facilitated the development of complex biological molecules capable of modifying intercellular signaling pathways using a targeted approach not previously achieved with chemically synthesized drugs [1]. These innovations brought a class of biopharmaceuticals that revolutionized the treatment paradigm for a wide range of chronic inflammatory diseases. Produced biologically with living cell-line cultures and recombinant DNA methods, monoclonal antibodies and fusion proteins that target Tumor Necrosis Factor alpha $(\mathrm{TNF} \alpha)$ and other pro-inflammatory mediators have been

\footnotetext{
Address correspondence to this author at the Department of Rheumatology, University of Algiers 1, EHS Ben Aknoun, 16306, Algiers, Algeria.,
}

E-mail address:ladjouze@hotmail.com shown to effectively control symptoms in patients with rheumatologic, gastroenterologic, and dermatologic inflammatory conditions who fail to respond to conventional first-line therapies [2 - 6]. However, the research and development costs for biologic agents are very high [7], as reflected in the market price, and, with increasing evidence and recognition of their effectiveness, they have become more extensively prescribed, leading to escalating healthcare costs [8].

Over the past decade, patent expiration for the anti-TNF $\alpha$ biologic agents adalimumab, etanercept, and infliximab, and the B cell-directed monoclonal antibody rituximab, created the opportunity for the development of "biosimilar" products, which are similar (but not identical) to the original biologics in protein structure, biologic activity, efficacy, and safety. Because research and development costs are lower for bio- 
similars than for original biologics, their introduction allows greater access to biologic treatment for more patients [9]. The World Health Organization (WHO) [10], the European Medicines Agency (EMA) in the European Union (EU) [11], and the Food and Drug Administration (FDA) in the United States (US) [12] have provided important guidance on biosimilarity and well-respected regulatory frameworks for biosimilar authorization. However, regulatory pathways and standards for biosimilars are still being formulated in many countries and, when available, may differ regionally and nationally. Moreover, in some countries, copies of reference products have been introduced without market authorization, without the guidance of experts, and without satisfying the rigorous regulations for biosimilars established to protect patient safety [13].

Many challenges posed by biosimilars are shared worldwide, while others may be specific to individual countries or regions. In the North African countries of Algeria, Morocco, and Tunisia, a regulatory framework for biosimilar approval remains in development. Few biosimilars for rheumatic diseases are currently available in North Africa and clinicians in this region have limited experience in prescribing them. Biosimilar use in clinical practice varies among Western countries and will likely also differ among countries in North Africa when these agents become more broadly available in the region. With limited biosimilar experience in North Africa, it is imperative that Healthcare Professionals (HCPs) gain a thorough understanding of these agents and the implications of their use. The objectives of this narrative review are to define and describe the various classes of currently available biopharmaceuticals; summarize current regulatory requirements and provide an update on approval, globally and in the North African region; and present key issues related to biosimilar use from the perspective of practicing rheumatologists in North Africa. Although many differences and similarities in clinical practice are evident between Western countries and North African countries, and may be of interest, such a comparison is beyond the scope of the current review.

\section{BIOPHARMACEUTICALS IN CHRONIC INFLAM- MATORY DISEASES: OVERVIEW}

\subsection{Background/Definitions}

Biologic agents are large molecules derived from living cells, usually manufactured using recombinant DNA or other biotechnologies. These agents (i.e., reference, originator, or innovator products) have greater complexity than chemically synthesized drugs because of their structural heterogeneity and specific activity within biologic systems, and their cost is usually higher $[5,14,15]$. Biologic Disease-Modifying AntiRheumatic Drugs (bDMARDs) such as anti-TNF $\alpha$ agents are very effective in the treatment of chronic immunoinflammatory diseases [4, 16, 17], but access is limited in many regions, including North Africa, partly because of cost constraints $[18,19]$.

Pharmaceutical companies are developing biosimilar versions for regulatory approval and introduction into global markets. Because of the natural inconsistencies in biologic sources and the unique, intricate (and usually proprietary) processes involved in engineering biologics, biosimilar manufacturers are unable to precisely replicate reference products [20]. Rigorous evaluation of biosimilars is thus required to establish their similarity to reference products in chemical structure, biologic activity, and clinical profile.

Although currently available in some countries, intended copies (i.e., non-comparable biologics or biomimics) are not supported by adequate evidence of comparability with reference products and fail to satisfy the requirements for biosimilarity established by regulatory authorities [13]. Certain intended copies have received regulatory approval based on generic drug standards [13].

\subsection{Regulatory Pathways}

Generic drugs are typically small, easily characterized molecules manufactured by chemical synthesis to produce exactly the same molecules as their respective reference products. Therefore, the demonstration of bioequivalence between generic and reference products in bioavailability studies is sufficient to assume therapeutic equivalence [10]. However, this approach is inappropriate for biosimilars as reference biologics are larger, more complex molecules that are more difficult to characterize and have clinical profiles that may be influenced by the manufacturing process. Consequently, biosimilar agents are not considered generics of biologics and are required to undergo more extensive investigation than generic drugs to obtain regulatory approval. To demonstrate biosimilarity, differences between biosimilar and reference products can be no greater than those expected between different batches of the same reference product and cannot affect the biosimilar's safety or efficacy.

In 2009, the WHO Expert Committee on Biological Standardization established global guidelines for evaluation and regulation of biosimilars $[10,21]$, which are reflected in a broad range of regional/national regulatory standards (Table 1). The WHO authorization process for biosimilars requires data from stepwise comparability exercises, including quality, nonclinical, and clinical studies. Similarly, for the EMA, comprehensive comparability studies must be conducted to demonstrate high similarity between the biosimilar and reference product in structure, biologic activity, safety, immunogenicity, and efficacy (Fig. 1) $[11,20]$. In the US, licensure by the FDA requires a robust characterization of the biosimilar based on a "totality of evidence" [22, 23], with sufficient clinical data to support the biosimilar's "safety, purity, and potency" when administered for one or more of the indications of the reference product. 


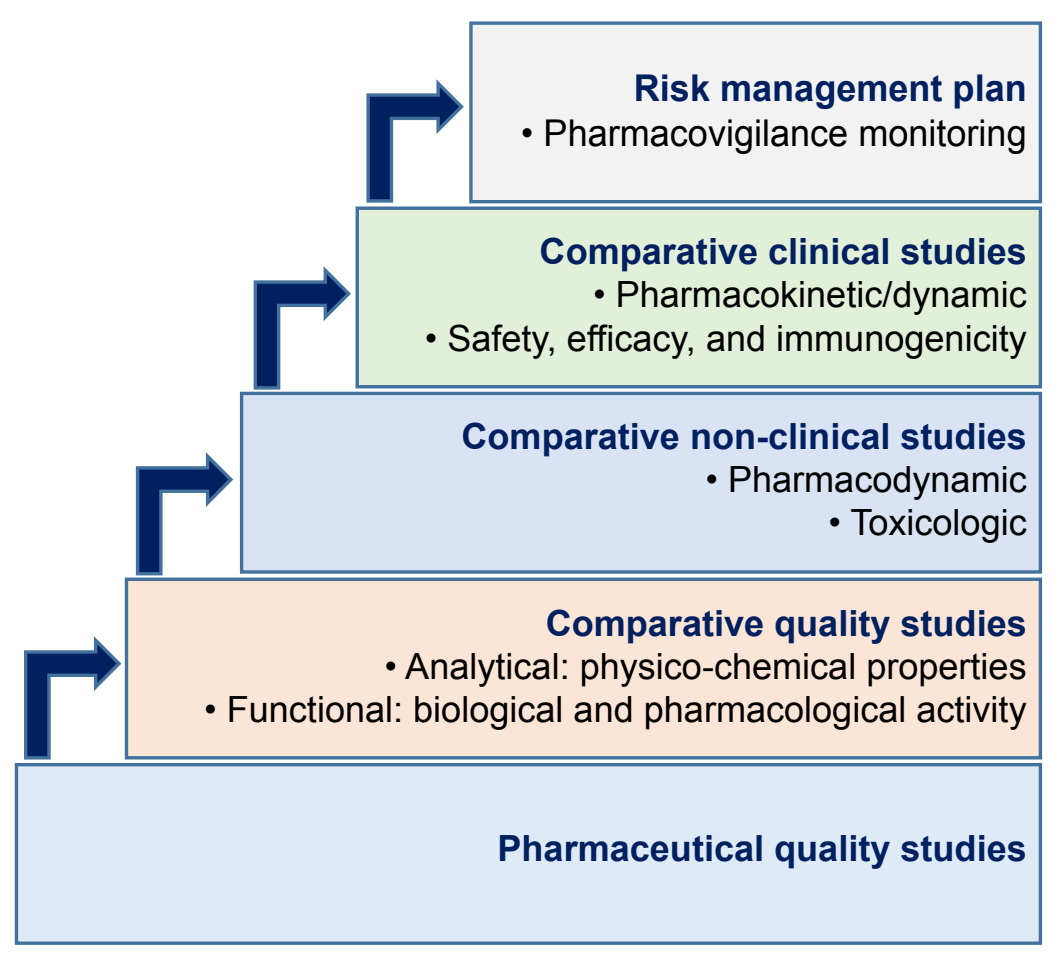

Fig. (1). Stepwise biosimilar development program required by European Medicines Agency guidelines [11,20].

Table 1. Summary of key regulatory pathways and requirements for biosimilar approval.

\begin{tabular}{|c|c|c|c|}
\hline \multirow[b]{2}{*}{ Requirement } & \multicolumn{3}{|c|}{ Regulatory authority } \\
\hline & $\mathrm{WHO}^{\mathrm{a}-\mathrm{c}}$ & $\operatorname{EMA}(\mathrm{EU})^{\mathrm{d}, \mathrm{e}}$ & FDA $(U S)^{f-i}$ \\
\hline $\begin{array}{c}\text { Approval pathway/ } \\
\text { development }\end{array}$ & $\begin{array}{l}\text { - Reference product (RP) must be licensed } \\
\text { in region/ country where biosimilar } \\
\text { approval is sought } \\
\text { • Biosimilar authorization is based on a } \\
\text { stepwise comparability exercise } \\
\text { o Quality studies: head-to-head } \\
\text { comparisons of quality and heterogeneity } \\
\text { (e.g., physicochemical, biological, } \\
\text { immunochemical properties); documented } \\
\text { manufacturing process; stability studies } \\
\text { o Non-clinical studies: Pharmacokinetic } \\
\text { (PK), Pharmacodynamic (PD), and } \\
\text { toxicological } \\
\text { o Clinical studies: clinical comparability } \\
\text { exercise (i.e., PK/PD studies followed by } \\
\text { pivotal clinical studies to show } \\
\text { comparable safety/effectiveness/ } \\
\text { immunogenicity) } \\
\text { • Varying amounts of data may be } \\
\text { requested by individual national regulatory } \\
\text { authorities } \\
\text { • Post-marketing surveillance is required }\end{array}$ & $\begin{array}{l}\text { - Abbreviated approval pathway } \\
\text { o EU-wide marketing authorization } \\
\text { granted via centralized EMA procedures } \\
\text { o Requires manufacturer submission of } \\
\text { a Marketing Authorization Application } \\
\text { (MAA) } \\
\text { o MAA evaluated by EMA's } \\
\text { Committee for Medicinal Products for } \\
\text { Human Use, Pharmacovigilance and } \\
\text { Risk Assessment Committee, and } \\
\text { Biologics/Biosimilar Working Parties } \\
\text { • Data required from stepwise } \\
\text { comprehensive comparability studies } \\
\text { o Pharmaceutical and comparative } \\
\text { quality studies } \\
\text { o Comparative non-clinical studies } \\
\text { o Comparative clinical studies of } \\
\text { safety/efficacy, PK/PD, and } \\
\text { immunogenicity } \\
\text { - If biosimilarity is shown, } \\
\text { safety/efficacy findings from clinical } \\
\text { trials of the RP may be used to support } \\
\text { MAA } \\
\text { o Allows for shorter and less costly } \\
\text { drug development program } \\
\text { • Post-marketing surveillance is required }\end{array}$ & $\begin{array}{l}\text { - Abbreviated approval pathway } \\
\text { o Created by the Biologics Price } \\
\text { Competition and Innovation Act under } \\
\text { the Affordable Care Act in } 2010 \\
(\$ 351[\mathrm{k}] \text {, Public Health Service Act) } \\
\text { o Requires manufacturer submission } \\
\text { of a Biologics License Application } \\
\text { (BLA) } \\
\text { o BLA evaluated by the FDA's Centers } \\
\text { for Drug/Biologics Evaluation and } \\
\text { Research } \\
\text { - Data required from } 3 \text { study types } \\
\text { o Analytical studies (e.g., } \\
\text { physicochemical, functional properties) } \\
\text { o Non-clinical studies (e.g., } \\
\text { toxicology) } \\
\text { o Clinical studies (e.g., PK, PD, } \\
\text { immunogenicity) } \\
\text { - If biosimilarity is shown, } \\
\text { safety/efficacy findings from clinical } \\
\text { trials of the RP may be used to support } \\
\text { BLA } \\
\text { o Allows for shorter and less costly } \\
\text { drug development program } \\
\text { - Post-marketing surveillance is } \\
\text { required }\end{array}$ \\
\hline Extrapolation & $\begin{array}{l}\text { - If biosimilarity is shown, the biosimilar } \\
\text { may obtain approval for other clinical } \\
\text { indications of the RP, even if not directly } \\
\text { assessed in clinical trials } \\
\text { - Scientific justification required }\end{array}$ & $\begin{array}{l}\text { - If biosimilarity is shown, the } \\
\text { biosimilar may obtain approval for other } \\
\text { clinical indications of the RP, even if not } \\
\text { directly assessed in clinical trials } \\
\text { - Scientific justification required }\end{array}$ & $\begin{array}{l}\text { - If biosimilarity is shown, the } \\
\text { biosimilar may obtain approval for } \\
\text { other clinical indications of the RP, } \\
\text { even if not directly assessed in clinical } \\
\text { trials } \\
\text { - Scientific justification required }\end{array}$ \\
\hline
\end{tabular}


(Table 1) contd.....

\begin{tabular}{|c|c|c|c|}
\hline & \multicolumn{3}{|c|}{ Regulatory authority } \\
\hline Requirement & \multirow{2}{*}{$\begin{array}{l}\text { - No guidance } \\
\text { - Practices to be defined by national } \\
\text { authorities }\end{array}$} & \multirow{2}{*}{$\begin{array}{l}\text { - No guidance } \\
\text { - Practices to be regulated by legislation } \\
\text { in individual EU countries }\end{array}$} & \multirow{2}{*}{$\begin{array}{l}\text { - Interchangeability requires data from } \\
3 \text { transitions from reference to } \\
\text { biosimilar product } \\
\text { - Switching requires data from } 1 \\
\text { transition from RP to biosimilar }\end{array}$} \\
\hline $\begin{array}{l}\text { Interchangeability/ } \\
\text { switching/ substitution }\end{array}$ & & & \\
\hline Nomenclature & $\begin{array}{l}\text { - Reference and related biosimilar } \\
\text { products share same nonproprietary name } \\
\text { (i.e., the International Nonproprietary } \\
\text { Name [INN]) } \\
\text { - Reference and related biosimilar } \\
\text { products will have unique "biological } \\
\text { qualifier" (BQ) added to the INN (2015 } \\
\text { proposal) } \\
\text { o BQ = 4 random lower-case consonants } \\
\text { - Example (fictitious) } \\
\text { o SBP = replicamab-jnzt } \\
\text { o RBP = replicamab-kngx }\end{array}$ & $\begin{array}{l}\text { - Reference and related biosimilar } \\
\text { products have distinct proprietary names } \\
\text { - Reference and related biosimilar } \\
\text { products share same nonproprietary } \\
\text { name (i.e., INN) } \\
\text { - Example (actual) } \\
\text { o Biosimilar = Remsima }{ }^{\circledR} \text { (infliximab) } \\
\text { o RP = Remicade }{ }^{\circledR} \text { (infliximab) } \\
\text { - Proprietary names and batch numbers } \\
\text { should appear on product packaging }\end{array}$ & $\begin{array}{l}\text { - Reference and related biosimilar } \\
\text { products have distinct proprietary } \\
\text { names } \\
\text { - Reference and related biosimilar } \\
\text { products have a non-proprietary proper } \\
\text { name that combines a shared core } \\
\text { name plus a unique suffix } \\
\text { o Core name = the name selected by } \\
\text { the US Pharmacopeial Convention for } \\
\text { the active substance (same for } \\
\text { biosimilar and RP) } \\
\text { o Suffix = } 4 \text { random lower-case letters } \\
\text { attached to the core name by a hyphen } \\
\text { (distinct for biosimilar and RP) } \\
\text { - Example (fictitious) } \\
\text { o Biosimilar = replicamab-jnzt } \\
\text { o RP = replicamab-kngx }\end{array}$ \\
\hline
\end{tabular}

${ }^{a}$ Reference [10]. ${ }^{b}$ Reference [21]. ${ }^{c}$ Reference [44]. ${ }^{\mathrm{d}}$ Reference [11]. ${ }^{\mathrm{e}}$ Reference [20]. ${ }^{\mathrm{f}}$ Reference [12]. ${ }^{\mathrm{g}}$ Reference [22]. ${ }^{\mathrm{h}}$ Reference [23]. ${ }^{\mathrm{i}}$ Reference [45]. ${ }^{\mathrm{j}}$ Reference [48]. EMA: European Medicines Agency; EU: European Union; US FDA: United States Food and Drug Administration; RP: reference product; WHO: World Health Organization.

\subsection{Currently Approved Biosimilars for Chronic Inflammatory Diseases (EU/US)}

Several biosimilars of the reference products adalimumab, etanercept, infliximab, and rituximab have been approved by the EMA or FDA, and many others are currently under review or in development (Table 2). In addition, biosimilars or intended copies of these reference agents are marketed globally or are in a pre-market development phase outside the EU and US (Table 3).

\section{BIOSIMILARS FOR CHRONIC INFLAMMATORY DISEASES IN NORTH AFRICA}

Countries in North Africa, including Algeria, Morocco, and Tunisia, have their own laws/regulations concerning registration of new pharmaceutical products but have not yet established legal/regulatory frameworks to help guide use of biosimilar products. In addition, no regional agency currently exists to provide overarching guidance on their regulation. In May 2018, the African Union adopted a treaty to establish the African Medicines Agency, but several important steps remain to be taken before this agency becomes a reality [24]. Indeed, a consensus on regulations may be difficult to achieve in this region because of between-country differences in healthcare systems and policies. Despite ongoing interest in these innovative agents, relatively few biosimilars are currently available for chronic inflammatory diseases in the region. North African regulatory authorities have not rushed biosimilar approvals as they assemble the necessary regulatory framework and await applications for suitable biosimilars.

\subsection{Algeria}

Biosimilar legislation is expected to be introduced soon in Algeria, based largely on the work of the country's expert committee in rheumatology. Launched in 2017 to oversee new product registration and approval, the National Agency of Pharmaceutical Products (ANPP) includes several expert committees of different specialties assembled to examine biosimilar registration applications. Relying in part on the recommendations of the WHO, EMA, and FDA, the ANPP will confirm that registered products satisfy biosimilarity, quality, efficacy, and safety standards; scrutinize manufacturing/marketing sites; and review post-marketing pharmacovigilance plans.

The Algerian Ministry of Health, Population, and Hospital Reform (MSPRH) routinely schedules meetings to allow review and discussion of product dossiers (including information on manufacturing, clinical/non-clinical study findings, and pharmacovigilance monitoring) by expert committee members. Meetings on biopharmaceuticals for rheumatic diseases include rheumatologists and other specialists involved in managing approved biologic/biosimilar products in their fields. The committee sends decisions regarding product approval/rejection to the Directorate of Pharmacy of the MSPRH, which makes the final decision and subsequently notifies applicants.

Biosimilars used in hematology, oncology, diabetology, and endocrinology have already been introduced in Algeria. In rheumatology, although several dossiers have been submitted, only a biosimilar of infliximab $\left(\right.$ Remsima $\left.^{\circledR}\right)$ has received marketing authorization (in 2017). 
Table 2. Biosimilar approval status in the European Union (EU) and/or United States (US) for chronic inflammatory diseases.

\begin{tabular}{|c|c|c|c|c|c|c|c|}
\hline \multirow{3}{*}{$\begin{array}{l}\text { Biologic } \\
\text { reference } \\
\text { product }\end{array}$} & \multirow{3}{*}{$\begin{array}{c}\text { Biosimilar } \\
\text { product } \\
\text { (proprietary } \\
\text { name) }\end{array}$} & \multicolumn{6}{|c|}{ Regulatory authority } \\
\hline & & \multicolumn{3}{|c|}{ EMA } & \multicolumn{3}{|c|}{ FDA } \\
\hline & & $\begin{array}{c}\text { Status } \\
\text { (date }[\text { month/year }] \text { ) }\end{array}$ & Company & Indication & $\begin{array}{c}\text { Status } \\
\text { (date [month/year]) }\end{array}$ & Company & Indication \\
\hline \multirow{11}{*}{$\begin{array}{l}\text { Adalimumab } \\
\left(\text { Humira }^{B}\right)^{a}\end{array}$} & $\begin{array}{c}\text { ABP 501 } \\
\text { (Amgevita }^{\circledR} \\
{[\text { EU/US]; }} \\
\text { Solymbic } \\
{[\text { EU]) }}\end{array}$ & $\begin{array}{l}\text { Approved } \\
(01 / 2017)\end{array}$ & Amgen & $\begin{array}{c}\text { CD, HS, JIA, } \\
\text { Ps, PsA, RA, } \\
\text { UC, uveitis }\end{array}$ & Approved (09/2016) & Amgen & $\begin{array}{c}\text { AS, CD, JIA, } \\
\text { Ps, PsA, RA, } \\
\text { UC }\end{array}$ \\
\hline & $\begin{array}{c}\text { BI 695501 } \\
\text { (Cyltezo }^{\circledR} \\
\text { [US]) }\end{array}$ & $\begin{array}{l}\text { Approved } \\
(11 / 2017)\end{array}$ & $\begin{array}{l}\text { Boehringer } \\
\text { Ingelheim }\end{array}$ & - & Approved (08/2017) & $\begin{array}{l}\text { Boehringer } \\
\text { Ingelheim }\end{array}$ & $\begin{array}{c}\text { AS, CD, JIA, } \\
\text { Ps, PsA, RA, } \\
\text { UC }\end{array}$ \\
\hline & $\begin{array}{c}\text { SB5 } \\
\left(\text { Imraldi }^{\circledR}\right)\end{array}$ & $\begin{array}{l}\text { Approved } \\
(06 / 2016)\end{array}$ & Samsung Bioepis & $\begin{array}{c}\text { CD, HS, JIA, } \\
\text { Ps, PsA, RA, } \\
\text { UC, uveitis }\end{array}$ & - & - & - \\
\hline & FKB327 & $\begin{array}{c}\text { MAA submitted } \\
(05 / 2017)\end{array}$ & $\begin{array}{l}\text { Fujifilm Kyowa } \\
\text { Kirin Biologics }\end{array}$ & - & - & - & - \\
\hline & GP2017 & $\begin{array}{l}\text { MAA submitted } \\
\quad(06 / 2017)\end{array}$ & Sandoz & - & - & - & - \\
\hline & ONS-3010 & $\begin{array}{c}\text { In phase III } \\
\text { development }\end{array}$ & Oncobiologics & - & $\begin{array}{c}\text { In phase III } \\
\text { development }\end{array}$ & Oncobiologics & - \\
\hline & PF-06410293 & - & - & - & $\begin{array}{c}\text { In phase III (RA) } \\
\text { development }\end{array}$ & Pfizer & - \\
\hline & M923 & - & - & - & $\begin{array}{l}\text { In phase III (Ps) } \\
\text { development } \\
\text { (positive results } \\
\text { reported in 2016) }\end{array}$ & $\begin{array}{l}\text { Momenta Pharma- } \\
\text { ceuticals }\end{array}$ & - \\
\hline & CHS-1420 & - & - & - & $\begin{array}{l}\text { In phase III (Ps) } \\
\text { development } \\
\text { (positive results } \\
\text { reported in 2017) }\end{array}$ & Coherus Biosciences & - \\
\hline & N/A & $\begin{array}{l}\text { In preclinical } \\
\text { development }\end{array}$ & $\begin{array}{c}\text { Adello } \\
\text { Biologics }\end{array}$ & - & $\begin{array}{l}\text { In preclinical } \\
\text { development }\end{array}$ & $\begin{array}{c}\text { Adello } \\
\text { Biologics }\end{array}$ & - \\
\hline & N/A & $\begin{array}{c}\text { In } \\
\text { pipeline }\end{array}$ & $\begin{array}{l}\text { AET BioTech/ } \\
\text { BioXpress } \\
\text { Therapeutics }\end{array}$ & - & - & - & - \\
\hline \multirow{4}{*}{$\begin{array}{l}\text { Etanercept } \\
\left(\text { Enbrel }^{\mathbb{B}}\right)^{\mathrm{b}}\end{array}$} & $\begin{array}{c}\text { SB4 } \\
\left(\text { Benepali }^{(\mathbb{R})}\right.\end{array}$ & $\begin{array}{l}\text { Approved } \\
(01 / 2016)\end{array}$ & Samsung Bioepis & $\begin{array}{c}\text { axSpA, JIA, } \\
\text { Ps, ped Ps, } \\
\text { PsA, RA }\end{array}$ & - & - & - \\
\hline & $\begin{array}{l}\text { GP2015 } \\
\left(\text { Erelzi }^{(\mathbb{R})}\right)\end{array}$ & $\begin{array}{l}\text { Approved } \\
(07 / 2017)\end{array}$ & Sandoz & $\begin{array}{c}\text { axSpA, JIA, } \\
\text { Ps, ped Ps, } \\
\text { PsA, RA }\end{array}$ & Approved (08/2016) & Sandoz & $\begin{array}{c}\text { axSpA, JIA, } \\
\text { Ps, ped Ps, } \\
\text { PsA, RA }\end{array}$ \\
\hline & $\begin{array}{l}\text { CHS-0214/B } \\
\text { AX } 2200 \text { [B] }\end{array}$ & - & - & - & $\begin{array}{c}\text { Global phase III trials: } \\
\text { Ps (RaPsODY) and } \\
\text { RA }\end{array}$ & $\begin{array}{c}\text { Coherus/ } \\
\text { Baxalta (US) }\end{array}$ & - \\
\hline & $\begin{array}{c}\mathrm{BX} 2922 \\
{[\mathrm{~B} / \mathrm{IC}]}\end{array}$ & In development & $\begin{array}{c}\text { BioXpress } \\
\text { Therapeutics } \\
\text { (Switzerland) }\end{array}$ & - & - & - & - \\
\hline \multirow{3}{*}{$\begin{array}{c}\text { Infliximab } \\
\left(\text { Remicade }^{\mathbb{B}}\right)^{\mathrm{c}}\end{array}$} & $\begin{array}{c}\text { CT-P13 } \\
\text { (Remsima }^{\circledR} \\
\text { [EU]; } \\
\text { Inflectra }^{\circledR} \\
\text { [US]) } \\
\end{array}$ & $\begin{array}{l}\text { Approved } \\
(09 / 2013)\end{array}$ & Celltrion & $\begin{array}{c}\text { AS, CD, Ps, } \\
\text { PsA, RA, UC }\end{array}$ & Approved (04/2016) & Hospira & $\begin{array}{c}\text { AS, CD, Ps, } \\
\text { PsA, RA, } \\
\text { UC }\end{array}$ \\
\hline & $\begin{array}{c}\text { SB2 } \text { (Flixabi }^{\circledR} \\
\text { [EU]; } \\
\text { Renflexis }^{\circledR} \\
\text { [US]) }\end{array}$ & $\begin{array}{l}\text { Approved } \\
(05 / 2016)\end{array}$ & Samsung Bioepis & $\begin{array}{c}\text { AS, CD, Ps, } \\
\text { PsA, RA, UC }\end{array}$ & Approved (04/2017) & Merck & $\begin{array}{c}\text { AS, CD, Ps, } \\
\text { PsA, RA, } \\
\text { UC }\end{array}$ \\
\hline & $\begin{array}{c}\text { PF-06438179 } \\
\text { (IXIFI }^{\mathrm{TM}} \text { ) }\end{array}$ & $\begin{array}{l}\text { Approved } \\
(12 / 2017)\end{array}$ & Pfizer & $\begin{array}{c}\text { AS, CD, Ps, } \\
\text { PsA, RA, UC }\end{array}$ & $\begin{array}{c}\text { Approval } \\
\text { recommended } \\
(03 / 2018)\end{array}$ & Sandoz & $\begin{array}{c}\mathrm{AS}, \mathrm{CD}, \mathrm{Ps} \\
\text { PsA, RA, } \\
\text { UC }\end{array}$ \\
\hline
\end{tabular}




\begin{tabular}{|c|c|c|c|c|c|c|c|}
\hline & STI-002 & - & - & - & $\begin{array}{c}\text { Phase III study in RA } \\
(05 / 2016)\end{array}$ & $\begin{array}{c}\text { Sorrento } \\
\text { Therapeutics (US) }\end{array}$ & - \\
\hline & NI-071 & - & - & - & $\begin{array}{l}\text { Phase III study in RA } \\
\text { (RADIANCE; } \\
\text { completion: } 12 / 2018 \text { ) }\end{array}$ & Sagent (US) & - \\
\hline & N/A & In development & $\begin{array}{l}\text { BioXpress } \\
\text { Therapeutics } \\
\text { (Switzerland) }\end{array}$ & - & - & - & - \\
\hline & ABP 710 & - & - & - & $\begin{array}{c}\text { In development } \\
\text { (positive results of } \\
\text { functional similarity } \\
\text { tests reported in 2017) }\end{array}$ & Amgen (US) & - \\
\hline \multirow{9}{*}{$\begin{array}{c}\text { Rituximab } \\
\left(\text { MabThera }^{\circledR /}\right. \\
\left.\text { Rituxan }^{\mathbb{B}}\right)^{\mathrm{d}}\end{array}$} & $\begin{array}{c}\text { CT-P10 } \\
\text { (Blitzima/ } \\
\text { Ritemvia/Tru } \\
\text { xima [EU]) }\end{array}$ & $\begin{array}{l}\text { Approved } \\
(07 / 2017)\end{array}$ & Celltrion & RA & $\begin{array}{l}\text { BLA submitted } \\
(06 / 2017)\end{array}$ & Teva/Celltrion & RA \\
\hline & APB 798 & - & - & - & $\begin{array}{l}\text { In phase III (RA) } \\
\text { development }\end{array}$ & $\begin{array}{l}\text { Amgen/ } \\
\text { Allergan }\end{array}$ & - \\
\hline & APO-RITUX & - & - & - & $\begin{array}{l}\text { In phase III (RA) } \\
\text { development }\end{array}$ & $\begin{array}{c}\text { Apotex } \\
\text { (Apobiologix; } \\
\text { Canada) }\end{array}$ & - \\
\hline & PF-05280586 & - & - & - & $\begin{array}{l}\text { In phase III } \\
\text { development }\end{array}$ & Pfizer (US) & - \\
\hline & $\begin{array}{l}\text { GP2013 } \\
\text { (Rixathon/ } \\
\text { Riximyo) }\end{array}$ & $\begin{array}{l}\text { Approved } \\
(06 / 2017)\end{array}$ & $\begin{array}{c}\text { Sandoz } \\
\text { (Switzerland) }\end{array}$ & RA & $\begin{array}{l}\text { BLA rejected } \\
(05 / 2018)\end{array}$ & - & - \\
\hline & $\mathrm{N} / \mathrm{A}[\mathrm{B}]$ & In development & $\begin{array}{l}\text { BioXpress } \\
\text { Therapeutics } \\
\text { (Switzerland) }\end{array}$ & - & - & - & - \\
\hline & JHL1101 & In development & JHL Biotech (China) & & - & - & - \\
\hline & $\mathrm{N} / \mathrm{A}[\mathrm{B}]$ & In development & $\begin{array}{l}\text { Mabion (Poland)/ } \\
\text { Mylan (Ireland) }\end{array}$ & - & - & - & - \\
\hline & N/A & In development & $\begin{array}{l}\text { Richter (Hungary)/ } \\
\text { Stada (Germany) }\end{array}$ & - & - & - & - \\
\hline
\end{tabular}

${ }^{\mathrm{a}}$ Reference [49]. ${ }^{\mathrm{b}}$ Reference [50]. ${ }^{\mathrm{c}}$ Reference [51]. ${ }^{\mathrm{d}}$ Reference [52].

AS: Ankylosing Spondylitis; axSpA: axial spondyloarthritis (non-radiographic axSpA and AS); [B]: Biosimilar; BLA: Biologics License Application; CD: Crohn's disease; EMA: European Medicines Agency; FDA: US Food and Drug Administration; HS: hidradenitis suppurative; [IC]: Intended Copy; JIA: Juvenile Idiopathic Arthritis; MAA: Marketing Authorization Application; N/A: Not Available; ped Ps: pediatric psoriasis (plaque); Ps: psoriasis (plaque); PsA: psoriatic arthritis; RA: Rheumatoid Arthritis; UC: Ulcerative Colitis..

\subsection{Morocco}

In the absence of laws or regulations on the use of biosimilars or intended copies, the Moroccan Drug and Pharmacy Directorate approves registered agents for chronic rheumatic diseases based on the opinion of an expert committee, including rheumatology/internal medicine pro-fessors and pharmacists. These experts examine evidence related to the product's manufacturing, structure and biological activity, safety/efficacy demonstrated in clinical studies, and plans for post-marketing pharmacovigilance. The Directorate's assessment is sent to the Moroccan Ministry of Health, the body responsible for final decisions regarding approval and pricing. In 2015, the Ministry of Health indicated that biosimilars are a better alternative for patients from a financial standpoint without providing more specific guidance. Remsima ${ }^{\circledR}$ is currently the only biosimilar authorized in Morocco.

The national safety institution and public/private insurance companies in Morocco currently provide reimbursement for biologic therapies according to treatment guidelines published for rheumatoid arthritis and ankylosing spondylitis, and as established by vthe Moroccan Society of Rheumatology $[25,26]$. In these guidelines, biosimilars are mentioned as
TNF-inhibitor options, with the same indications as the reference products, but no switching recommendations are offered.

\subsection{Tunisia}

The Tunisian Directorate of Pharmacy and Medicine is ultimately responsible for regulatory submissions and authorization of new products in Tunisia. The assessment process is multi-layered, involving the National Medicine Control Laboratory, specialized scientific commissions, and the Technical Committee for Proprietary Medicinal Products. With national regulations on biosimilars lacking, the Tunisian authorities apply procedures recommended by the WHO, EMA, and FDA. Several biosimilars for other diseases have been approved in Tunisia (e.g., erythropoietin, filgrastim, and somatropin biosimilars). Remsima ${ }^{\circledR}$ was the first biosimilar for rheumatic disease to be introduced in Tunisia, generating discussion about its possible exclusive use given the country's current socioeconomic situation. Tunisian authorities recently revealed plans to approve the least expensive product, i.e., Remsima $^{\circledR}$ or Remicade ${ }^{\circledR}$. 
Table 3. Biosimilars and intended copies approved, or in development, outside of the European Union/United States (US) for chronic inflammatory diseases.

\begin{tabular}{|c|c|c|c|c|}
\hline $\begin{array}{l}\text { Biologic reference } \\
\text { product }\end{array}$ & $\begin{array}{c}\text { Biosimilar [B] / } \\
\text { intended copy [IC] } \\
\text { (proprietary name) } \\
\end{array}$ & $\begin{array}{c}\text { Status } \\
\text { (date }[\text { month/year] })\end{array}$ & Company & Indication \\
\hline \multirow{9}{*}{$\begin{array}{l}\text { Adalimumab } \\
\left(\text { Humira }^{\mathbb{B}}\right)^{\mathrm{a}}\end{array}$} & ZRC3197 [IC] (Exemptia) & Approved in India $(12 / 2014)$ & Zydus Cadila (India) & RA \\
\hline & Adfrar [IC] & Approved in India $(01 / 2016)$ & Torrent Pharmaceuticals (India) & $\begin{array}{l}\text { AS, Ps, PsA, } \\
\text { RA, UC }\end{array}$ \\
\hline & ONS-3010 [B/IC] & In phase III development & GMS Tenshi (China, India, Mexico) & - \\
\hline & $\mathrm{LBAL}[\mathrm{B} / \mathrm{IC}]$ & In phase III (RA) development & $\begin{array}{c}\text { LG Life Sciences (Korea)/Mochida } \\
\text { Pharmaceutical (Japan) }\end{array}$ & - \\
\hline & BCD 057 [B/IC] & $\begin{array}{c}\text { In phase III (Ps, RA) } \\
\text { development }\end{array}$ & Biocad (Russia) & - \\
\hline & PBP1502 [B/IC] & In phase I development & Prestige BioPharma (Singapore) & - \\
\hline & N/A [B/IC] & In development & $\begin{array}{c}\text { PlantForm (Canada)/ } \\
\text { Axis Biotec Brasil (Brazil) }\end{array}$ & - \\
\hline & $\mathrm{N} / \mathrm{A}[\mathrm{B} / \mathrm{IC}]$ & In preclinical development & mAbxience (Spain) & - \\
\hline & CT-P17 & In pipeline & Celltrion (Korea) & - \\
\hline \multirow{12}{*}{$\begin{array}{l}\text { Etanercept } \\
\left(\text { Enbrel }^{\mathbb{B}}\right)^{\mathrm{b}}\end{array}$} & $\begin{array}{c}\text { SB4 [B] } \\
\left(\text { Brenzys }^{\circledR}\right)\end{array}$ & $\begin{array}{c}\text { Approved in South Korea } \\
(09 / 2015), \text { Australia }(07 / 2016) \\
\text { Canada }(08 / 2016)\end{array}$ & Samsung Bioepis (Korea)/MSD (US) & $\begin{array}{l}\operatorname{axSpA}, \text { JIA, } \\
\text { ped Ps, Ps, } \\
\text { PsA, RA }\end{array}$ \\
\hline & $\begin{array}{l}\text { GP2015 [B] } \\
\quad\left(\text { Erelzi }^{(\mathbb{B})}\right)\end{array}$ & Approved in Canada $(08 / 2017)$ & Sandoz (Switzerland) & $\begin{array}{l}\operatorname{axSpA}, \text { JIA, } \\
\text { ped Ps, Ps, } \\
\text { PsA, RA }\end{array}$ \\
\hline & $\begin{array}{l}\text { HD203 [B] } \\
\text { (Davictrel) }\end{array}$ & Approved in Korea $(11 / 2014)$ & Hanwha Chemical (Korea) & $\begin{array}{l}\text { AS, RA, Ps, } \\
\text { PsA }\end{array}$ \\
\hline & LBEC0101 [B] & $\begin{array}{l}\text { Phase III trials ongoing in RA; } \\
\text { filed for approval in Japan }\end{array}$ & $\begin{array}{c}\text { LG Life Sciences (Korea)/Mochida } \\
\text { Pharmaceutical (Japan) }\end{array}$ & - \\
\hline & $\begin{array}{l}\text { ENIA11 [B] } \\
\left(\text { TuNEX }^{\mathbb{B}}\right)\end{array}$ & $\begin{array}{c}\text { Registered (RA; Taiwan); Phase } \\
\text { III trials in RA/AS (Japan, } \\
\text { Korea) }\end{array}$ & $\begin{array}{c}\text { Mycenax Biotech/ } \\
\text { TSH Biopharm (Taiwan) }\end{array}$ & - \\
\hline & CT-P05 [B/IC] & In development & Celltrion (Korea) & - \\
\hline & AVGO1 [B/IC] $\left(\right.$ Avent $\left.{ }^{\mathrm{TM}}\right)$ & $\begin{array}{l}\text { Patented in India (2010); } \\
\text { similarity demonstrated in } \\
\text { preclinical trials }\end{array}$ & Avesthagen (India) & - \\
\hline & Intacept $^{\circledR}[\mathrm{B} / \mathrm{IC}]$ & Approved in India $(03 / 2015)$ & Intas Pharmaceuticals (India) & $\begin{array}{l}\text { AS, JIA, RA, } \\
\text { Ps, PsA }\end{array}$ \\
\hline & $\mathrm{N} / \mathrm{A}[\mathrm{B} / \mathrm{IC}]$ & In preclinical development & mAbxience (Spain) & - \\
\hline & PRX-106 [B/IC] & In preclinical development & Protalix Biotherapeutics (Israel) & - \\
\hline & $\begin{array}{c}\text { Etanar }^{(\mathbb{B}} / \text { Etart }^{(\mathbb{B}} / \text { Etacept }^{(\mathbb{B}} / \\
\text { Yisaipu }^{(}[\mathrm{IC}]\end{array}$ & $\begin{array}{c}\text { Approved in Colombia, Mexico, } \\
\text { India, China }\end{array}$ & $\begin{array}{l}\text { Shanghai CP Guojian (China) } \\
\text { Cipla (India) }\end{array}$ & $\begin{array}{l}\text { AS, RA, Ps } \\
\text { AS, JIA, RA, } \\
\text { Ps, PsA }\end{array}$ \\
\hline & Infinitam $^{\circledR}$ & Approved in Mexico & Probiomed (Mexico) & RA \\
\hline \multirow{6}{*}{$\begin{array}{l}\text { Infliximab } \\
\text { (Remicade) }^{\mathrm{c}}\end{array}$} & $\begin{array}{c}\text { CT-P13 [B/IC }] \\
\text { (Remsima/Inflectra/others) }\end{array}$ & $\begin{array}{l}\text { Approved in } 79 \text { countries } \\
\text { (as of } 01 / 2017 \text { ) }\end{array}$ & Celltrion & $\begin{array}{c}\text { AS, CD, Ps, } \\
\text { PsA, } \\
\text { RA, UC }\end{array}$ \\
\hline & SB2 [B] (Renflexis) & $\begin{array}{l}\text { Approved in Korea }(12 / 2015), \\
\text { Australia }(11 / 2016)\end{array}$ & Samsung Bioepis/MSD (Korea/US) & $\begin{array}{c}\text { AS, CD, Ps, } \\
\text { PsA, } \\
\text { RA, UC }\end{array}$ \\
\hline & Infliximab BS [B/IC] & Approved in Japan $(07 / 2014)$ & Nippon Kayaku (Japan) & $\mathrm{CD}, \mathrm{RA}, \mathrm{UC}$ \\
\hline & BOW015 [IC] (Infimab) & $\begin{array}{c}\text { Approved in India }(09 / 2014) \text {; } \\
\text { global phase III study initiated in } \\
\text { RA }(02 / 2016)\end{array}$ & $\begin{array}{l}\text { Ranbaxy Laboratories/Epirus } \\
\text { Biopharmaceuticals (India/US) }\end{array}$ & $\begin{array}{c}\mathrm{AS}, \mathrm{CD}, \mathrm{Ps}, \\
\text { PsA, } \\
\text { RA, CD } \\
\end{array}$ \\
\hline & NI-071 [B/IC] (Nichi-Iko) & Approved in Japan $(09 / 2017)$ & Nichi-Iko/Zeria (Japan) & - \\
\hline & STI-002 [B/IC] & $\begin{array}{l}\text { Positive phase III study findings } \\
\text { in RA reported }(05 / 2016)\end{array}$ & $\begin{array}{c}\text { MabTech/Sorrento Therapeutics } \\
\text { (China/US) }\end{array}$ & - \\
\hline
\end{tabular}




\begin{tabular}{|c|c|c|c|c|}
\hline \multirow{11}{*}{$\begin{array}{c}\text { Rituximab } \\
{\text { (MabThera/Rituxan })^{\mathrm{d}}}^{\text {(Mab) }}\end{array}$} & CT-P10 (Truxima) & Approved in Korea $(11 / 2016)$ & Celltrion & RA \\
\hline & $\begin{array}{l}\text { BCD-020 (Acellbia [Russia/India]; } \\
\text { USMAL [Bolivia/Honduras]) }\end{array}$ & $\begin{array}{l}\text { Approved in Russia, Bolivia, } \\
\text { Honduras } \\
\text { Pre-registration for RA in India } \\
\text { (2017) }\end{array}$ & Biocad (Russia) & \\
\hline & Reditux & $\begin{array}{c}\text { Approved in Bolivia, Chile, } \\
\text { Ecuador, Paraguay, Peru, India } \\
(2007)\end{array}$ & Dr. Reddy’s Laboratories & RA \\
\hline & Novex & Approved in Argentina (2013) & $\begin{array}{c}\text { mAbxience/Laboratorio Elea } \\
\text { (Spain/Argentina) }\end{array}$ & RA \\
\hline & Kikuzubam & $\begin{array}{l}\text { Approved in Bolivia, Chile, } \\
\text { Mexico, Peru }\end{array}$ & Probiomed (Mexico) & RA \\
\hline & MabTas & Approved in India (2013) & Intas Biopharmaceuticals (India) & RA \\
\hline & N/A & Approved in India (2013) & Zenotech Laboratories (India) & RA \\
\hline & Maball & Approved in India (2015) & Hetero Group (India) & $\mathrm{RA}$ \\
\hline & N/A [IC] & In development & Torrent Pharmaceuticals (India) & - \\
\hline & HLX01 [IC] & In phase III development & Shanghai Henlius Biotech (China) & - \\
\hline & JHL1101 & In phase III development & JHL Biotech (China) & \\
\hline
\end{tabular}

${ }^{a}$ Reference [49]. ${ }^{\mathrm{b}}$ Reference [50]. ${ }^{\mathrm{c}}$ Reference [51]. ${ }^{\mathrm{d}}$ Reference [52]. ${ }^{\mathrm{e}}$ Reference [53]. AS: Ankylosing Spondylitis; axSpA: axial spondyloarthritis (non-radiographic axSpA and AS); CD: Crohn's disease; JIA: Juvenile Idiopathic Arthritis; ped Ps: pediatric psoriasis (plaque); Ps: psoriasis (plaque); PsA: psoriatic arthritis; RA: Rheumatoid Arthritis; UC: Ulcerative Colitis.

The Tunisian League Against Rheumatism (LITAR) and the Tunisian Gastroenterology Society have recommended that safe and effective treatments be made available to Tunisian patients at the lowest cost possible. However, all new medications must be introduced with caution to ensure patient safety. These societies are developing a biosimilar position paper based on clinical study findings, relevant policies of other rheumatology/gastroenterology societies, and the country's current economic circumstances. Biosimilar approval and use should be based on multiple factors, including safety, efficacy, and traceability/interchangeability. While the value of introducing biosimilars in Tunisia is recognized, a strict legal and regulatory framework must be in place and prescription of biologic/biosimilar products must remain a clinical decision, made in consultation with patients and in consideration of possible health consequences, safety concerns, and economic impact. LITAR does not approve switching a patient who is stable on a biotherapy to a biosimilar to reduce costs without prior consent of the prescriber and patient. Biologic-tobiosimilar switching requires careful consideration on a caseby-case basis, not automatic implementation. Interchangeability studies have demonstrated safety and efficacy when switching from reference product to biosimilar, but not the inverse. Moreover, the societies have reservations about the importation of products that have not undergone rigorous quality control, e.g., intended copies.

\section{ESSENTIAL CONCERNS: PERSPECTIVES OF NORTH AFRICAN RHEUMATOLOGISTS}

\subsection{Adherence to Established Regulatory Biosimilarity Standards}

Regulatory pathways for biosimilar approval by the EMA and FDA require the submission of data from head-to-head clinical studies carefully designed to rule out the possibility of clinically relevant differences between biosimilar and reference products $[11,23]$. Ideally, the pathways established by these large regulatory authorities will be universally adopted at regional and national levels around the world, including North Africa. Biosimilar approval based on international criteria has been shown to instill confidence among clinicians [27]. However, achievement of such harmonization will be challenging given the profound cultural/political/social/economic disparities among continents, countries, and regions.

In North Africa, as elsewhere, adoption of legislation defining the pathway for biosimilars should ideally precede establishment of the regulatory framework for the biosimilar approval process. Acceptance of a statutory basis for this process will help ensure that stakeholders have the opportunity to offer their input and expertise in developing and evaluating fundamental principles. Moreover, subsequent changes to regulation or guidance would need to be consistent with the statute, providing stakeholders with a stable, predictable regulatory environment.

Many North African rheumatologists are aware of the potential cost/access benefits of biosimilars but may not favor biosimilar authorization unless manufacturers have received authorization from other globally respected regulatory agencies. Intended copies with limited or non-comparable data are not expected to receive market authorization in this region because they do not satisfy stringent regulatory approval criteria and may pose a threat to patient safety.

\subsection{Biosimilar Data Availability and Comparability}

A substantial number of biosimilar products, including biosimilars of adalimumab, infliximab, etanercept, and rituximab, do not have published the evidence of structural and functional comparability from non-clinical studies [28]. Additionally, much of the evidence from registration studies has only been published in conference abstracts. Release of findings from biosimilar trials in the public domain as full-text publications is essential to ensure that HCPs and patients are well informed.

Clinicians may have safety concerns related to biosimilar immunogenicity, as small or indiscernible differences arising during production may result in antidrug antibody formation [28]. Differences in immunogenicity between biosimilars of 
monoclonal antibodies or fusion proteins and their reference products may be especially likely because of the large size and complexity of the reference biomolecules and post-translational modifications. Considerable differences in immunogenicity rates among biotherapies used to treat chronic inflammatory diseases have been reported in some clinical trials, with the highest rates seen with adalimumab, infliximab, and the infliximab biosimilar CT-P13 [29].

In a recent randomized controlled trial, Emery et al. observed a significant difference in the incidence of antidrug antibodies in patients treated with reference product versus biosimilar (SB4) etanercept, although this difference was not considered clinically relevant [30]. By contrast, in another study, the incidence of antidrug antibody development was consistent among patients receiving reference product and biosimilar (SB2) infliximab [31]. Immunogenicity can result in reduced therapeutic levels and loss of efficacy after months or years of treatment, requiring dose increases and/or shortening of dosing intervals in some patients. However, despite the frequency and clinical consequences of biologic immunogenicity, relatively limited data have been published on immunogenicity in biosimilar clinical trials.

The comparability of efficacy between biosimilars and anti-TNF $\alpha$ agents has also raised questions. In phase III comparative studies, biosimilars of reference TNF inhibitors satisfied pre-specified criteria for equivalence in efficacy in patients with rheumatoid arthritis [30] and plaque psoriasis [32]. However, higher treatment response rates were observed with both biosimilars and their reference biologics in recent comparative clinical trials than with the reference biologics in pivotal registration trials [33]. Although these differences in efficacy may have resulted from differences in study design and/or patient characteristics, the findings warrant additional research. Because definitive conclusions cannot be drawn from a comparison of findings from clinical trials with different designs and methodology, a strong argument can be made in favor of standardization of future biosimilar studies [34]. Greater uniformity across biosimilar clinical studies may also increase HCP confidence in these biopharmaceuticals.

\subsection{Interchangeability, Switching, and Substitution}

Future regulations for biosimilar approval in North Africa may include guidelines on interchangeability, switching, and substitution among biologic and biosimilar products. According to the EMA, interchangeability is defined as the possibility of replacing a reference product with a biosimilar (or the inverse) or replacing one biosimilar product with another [20]. The clinician may decide to exchange one product for another, with the same therapeutic intent, a practice known as "switching." Alternatively, one interchangeable product may be dispensed instead of another at the pharmacy level without consultation with, or the consent of, the prescriber, which is known as "substitution."

Because established regulatory authorities such as the WHO and EMA do not require switching studies for biosimilar approval and do not provide guidance on this practice (Table 1), the effects of switching are often not evaluated in biosimilar registration studies [35]. However, the FDA does require the submission of data on alternating (one transition) and switching (three transitions) to support these practices [36].

In a 2012 review, Ebbers et al. found no evidence of safety concerns related to switching from biologic to biosimilar agents in clinical studies or post-marketing surveillance [37]. Similarly, in a 2017 review, Moots et al. found no differences in safety/efficacy outcomes with or without switching between the reference biologics adalimumab, infliximab, etanercept, and rituximab and their biosimilars [35]. The authors nonetheless concluded that the available evidence is qualitatively and quantitatively insufficient to confirm the safety /efficacy of switching. In systematic literature reviews of randomized controlled trials and real-world studies, Numan et al. [38] and McKinnon et al. [39] reached similar conclusions with regard to non-medical switching, which occurs as a result of non-medical concerns such as treatment costs. The studies conducted in patients with chronic inflammatory diseases were not adequately designed to assess efficacy and safety after nonmedical switching, and evidence from these studies was often inconsistent and inconclusive. Even when assessing only the 17 randomized controlled trials found in their literature searches (January 2012 to February 2018; (Table 4), Numan et al. found that none satisfied all of the study design elements considered important for robust switching studies [38].

Given that the goals of switching from a stable biologic to a biosimilar are cost savings and broader access, because biosimilars have not demonstrated better safety or efficacy, regulatory agencies will need to determine whether they may be substituted for reference biologics for non-clinical reasons. To date, in the EU, no country has explicitly authorized the automatic substitution of products from different manufacturers without clinician/patient involvement. In many regions, including North Africa, pharmacy-level substitution is generally not considered appropriate unless stringent legal and regulatory criteria are satisfied in addition to biosimilarity requirements. Prescribing clinicians are also supported as primary decision-makers, with the right to prescribe the most appropriate product based on their clinical judgment, scientific evidence, and individual patient profiles, and to override automatic substitution not deemed in a patient's best interest.

\subsection{Pharmacovigilance}

Plans for post-marketing monitoring and risk-management activities, at least as rigorous as those for reference biologics, are essential for biosimilar approval [10, 11, 20, 23], and are expected to be mandatory in the legal/regulatory framework for biosimilar authorization in North Africa. As with all medications, adverse events related to biosimilar use in daily practice can only be detected through continuous post-approval surveillance at the clinical level [40]. The importance of postmarketing surveillance in identifying safety concerns related to intended copies was demonstrated with the rituximab intended copy Kikuzubam ${ }^{\circledR}$, as reports of anaphylactic reactions in rheumatology patients switched to this biosimilar in the Mexican pharmacovigilance program resulted in its removal from the market $[41,42]$. 
Table 4. Summary of randomized controlled non-medical switching studies in chronic inflammatory diseases [38].

\begin{tabular}{|c|c|c|c|c|}
\hline $\begin{array}{l}\text { Rheumatic } \\
\text { Disease }\end{array}$ & Study (Study Name) & $\begin{array}{l}\text { Biosimilar (n) Product [Switch Group] / } \\
\text { Biologic Reference Product (n) [Control } \\
\text { Group] }\end{array}$ & $\begin{array}{l}\text { Post-Switch } \\
\text { Follow-up } \\
\text { Duration, wk }\end{array}$ & $\begin{array}{c}\text { Patients Discontinuing Treatment, } \mathrm{n} \\
(\%) \\
\text { (Switch vs. Control Group) }\end{array}$ \\
\hline \multirow[t]{9}{*}{ RA } & $\begin{array}{l}\text { Cohen } \text { et al. } 2018 \text { [54] } \\
\text { (VOLTAIRE-RA) }\end{array}$ & BI695501 (n=147) / adalimumab $(\mathrm{n}=147)$ & $24-34$ & $9(6 \%)$ vs. $8(5 \%)$ \\
\hline & $\begin{array}{l}\text { Genovese } 2017 \text { [55] } \\
\text { (ARABESC-OLE) }\end{array}$ & FKB327 $(n=108) /$ adalimumab $(n=213)$ & 76 & NR \\
\hline & Weinblatt et al. 2017 [56] & SB5 $(n=125) /$ adalimumab $(n=129)$ & 28 & $8(6 \%)$ vs. $5(4 \%)$ \\
\hline & Cohen et al. $2017[57,58]$ & ABP $501(n=237) /$ adalimumab $(n=229)$ & 46 & $30(13 \%)$ vs. $25(11 \%)$ \\
\hline & Emery et al. 2017 [59] & SB4 $(n=119) /$ etanercept $(n=126)$ & 48 & $6(5 \%)$ vs. $7(6 \%)$ \\
\hline & Smolen et al. 2018 [31] & SB2 $(n=94) /$ infliximab $(n=101)$ & 16 & $6(6 \%)$ vs. $5(5 \%)$ \\
\hline & Tanaka et al. 2017 [60] & CT-P13 (n=33) / infliximab (n=38) & 105 & $11(33 \%)$ vs. $6(16 \%)$ \\
\hline & $\begin{array}{l}\text { Yoo et al. } 2017 \text { [61] } \\
\text { (PLANETRA) }\end{array}$ & CT-P13 (n=144) / infliximab $(\mathrm{n}=158)$ & 48 & $16(11 \%)$ vs. $25(16 \%)$ \\
\hline & Taylor et al. 2016 [62] & BOW015 (n=53) / infliximab (n=104) & 38 & NR \\
\hline AS & $\begin{array}{l}\text { Park et al. } 2017 \text { [63] } \\
\text { (PLANETAS) }\end{array}$ & CT-P13 (n=86) / infliximab (n=88) & 48 & $9(10 \%)$ vs. $7(8 \%)$ \\
\hline \multirow[t]{4}{*}{$\mathrm{Ps} \pm \mathrm{PsA}$} & $\begin{array}{l}\text { Blauvelt et al. } 2017 \text { [64] } \\
\text { (ADACCESS) }\end{array}$ & GP2017 $(n=63) /$ adalimumab $(n=127)$ & 34 & $16(25 \%)$ vs. $23(18 \%)$ \\
\hline & Hodge et al. 2017 [65] & CHS-1420 $(n=124) /$ adalimumab $(n=129)$ & 8 & NR \\
\hline & Papp et al. 2017 [66] & ABP $501(n=77) /$ adalimumab $(n=79)$ & 36 & $9(12 \%)$ vs. $8(10 \%)$ \\
\hline & $\begin{array}{c}\text { Griffiths et al. } 2017[32,67] \\
\text { (EGALITY) }\end{array}$ & GP2015 $(n=96) /$ etanercept $(n=151)$ & 40 & $6(6 \%)$ vs. $14(9 \%)$ \\
\hline $\begin{array}{l}\text { CD, UC, RA, } \\
\text { Ps, PsA, SpA }\end{array}$ & $\begin{array}{c}\text { Jørgensen et al. } 2017[68,69] \\
\text { (NOR-SWITCH) }\end{array}$ & CT-P13 $(n=240) /$ infliximab $(n=241)$ & 78 & $18(8 \%)$ vs. $25(10 \%)$ \\
\hline \multirow[t]{2}{*}{$\mathrm{IBD} / \mathrm{CD}$} & $\begin{array}{l}\text { Volkers et al. } 2017 \text { [70] } \\
\text { (SIMILAR) }\end{array}$ & CT-P13 (n=15) / infliximab $(n=6)$ & 30 & NR \\
\hline & Ye et al. $2018[71,72]$ & CT-P13 $(\mathrm{n}=55) /$ infliximab $(\mathrm{n}=54)$ & 24 & NR \\
\hline
\end{tabular}

AS: Ankylosing Spondylitis; CD: Crohn's Disease; IBD: Inflammatory Bowel Disease; NR: Not Reported; Ps: Psoriasis (plaque); PsA: Psoriatic Arthritis; RA: Rheumatoid Arthritis; SpA: Spondyloarthritis; UC: Ulcerative Colitis.

Over the past few decades, awareness of the importance of pharmacovigilance to the healthcare system in Africa has increased [43]. Morocco and Tunisia were among the first African member countries of the WHO International Drug Monitoring Programme (1992-1993), and Algeria subsequently became an associate member. In North Africa, postmarketing monitoring of drug safety is hindered by several common obstacles, including HCPs' lack of awareness of surveillance requirements and reporting forms, unfamiliarity with product labeling, inability to identify adverse events, and hesitation to report events because of guilt or fear of legal action. Improved HCP education/training and greater financial/logistical support for pharmacovigilance systems are needed to overcome these challenges.

\subsection{Biopharmaceutical Nomenclature}

Biosimilars require names that can be easily distinguished from those of reference biologics to ensure accurate identification and effective pharmacosurveillance, and to reduce the risk of unintended substitution of non-interchangeable products. Guidance on biologic and biosimilar names has been provided by the WHO, EMA, and FDA [20, 44, 45], as summarized in Table 1. To date, recommended approaches are inconsistent, which may lead to confusion, but further refinement is expected.

\subsection{Accessibility and Cost Issues}

Since the first biosimilar (Omnitrope ${ }^{\circledR}$ ) was approved in the EU in 2006, the biosimilar pipeline has seen remarkable growth. Introduction of biosimilar products is anticipated to reduce costs and expand patient access in public and private healthcare systems $[46,47]$. Although the difference in price between reference and biosimilar products may be $<30 \%$, such a cost saving is sufficient to generate considerable interest, particularly in countries with negative economic forecasts. In addition to wider access for patients of all socioeconomic levels, competition in biosimilars may lead to lower prices of the reference biologics. In countries facing a difficult economic situation, such as Tunisia, competitive pricing of reference biologics is particularly important.

Accessibility to healthcare and treatment is problematic across North Africa, but each country has specific challenges. In Algeria, the state provides free treatment for the entire population, regardless of socioeconomic status, but costs must remain below a specified threshold. In Morocco and Tunisia, patients covered by the social security system are entitled to receive free treatment. However, the number of patients requiring specialist care and biopharmaceuticals affects access, so that a cost reduction of only $20-30 \%$ for biosimilars is expected to improve access. Nonetheless, rheumatologists are cautious that lower cost for, and increased patient access to, biosimilars is not achieved at the expense of patient safety. 


\section{CONCLUSION}

Biosimilar versions of original biologic agents used in a wide range of chronic inflammatory diseases may allow greater patient access to treatment through cost savings. However, they pose several challenges, particularly in developing countries. In North Africa, work is underway to establish the legal/ regulatory framework for biosimilar authorization to ensure that patients and HCPs are protected. HCPs will require information about the regulatory pathways in place in their countries and the clinical profiles of the biosimilars authorized by their regulatory agencies. Their perspectives on switching and substitution require consideration, allowing for clinical decision-making on a case-by-case basis in alignment with scientific evidence and patient-, disease-, and product-specific factors.

\section{CONSENT FOR PUBLICATION}

Not applicable.

\section{FUNDING}

None.

\section{CONFLICT OF INTEREST}

O.M. has received honoraria for speaking engagements funded by AbbVie, Janssen, Novartis, Pfizer, and Roche. H.S.S. has participated in ad hoc speaking engagements for AbbVie, Fidia, Janssen, NewBridge, Pfizer, Roche, and Sanofi. S.K. has no financial interest or lasting/permanent associations; he has participated as an invited speaker in conferences sponsored by AbbVie, MSD, Pfizer, Roche, and Sanofi. Y.M.D. and S.A.-I.M. are employees and shareholders of Pfizer; M.M.A. was an employee and shareholder of Pfizer when the review was developed. S.L.-T. and A.L.-R. have no conflicts of interest to disclose.

\section{ACKNOWLEDGEMENTS}

Medical writing support was provided by Donna McGuire of Engage Scientific Solutions and was funded by Pfizer. The authors would also like to acknowledge Heather Jones of Pfizer, Collegeville, for her important contributions to the development of this review.

\section{REFERENCES}

[1] Croft M, Siegel RM. Beyond TNF: TNF superfamily cytokines as targets for the treatment of rheumatic diseases. Nat Rev Rheumatol 2017; 13(4): 217-33.

[http://dx.doi.org/10.1038/nrrheum.2017.22] [PMID: 28275260]

[2] Nam JL, Takase-Minegishi K, Ramiro S, et al. Efficacy of biological disease-modifying antirheumatic drugs: A systematic literature review informing the 2016 update of the EULAR recommendations for the management of rheumatoid arthritis. Ann Rheum Dis 2017; 76(6): 1113-36.

[http://dx.doi.org/10.1136/annrheumdis-2016-210713] [PMID: 2828 3512]

[3] Stidham RW, Lee TC, Higgins PD, et al. Systematic review with network meta-analysis: the efficacy of anti-TNF agents for the treatment of Crohn's disease. Aliment Pharmacol Ther 2014; 39(12): 1349-62.

[http://dx.doi.org/10.1111/apt.12749] [PMID: 24749763]

[4] Sbidian E, Chaimani A, Garcia-Doval I, et al. Systemic pharmacological treatments for chronic plaque psoriasis: A network meta-analysis. Cochrane Database Syst Rev 2017; 12CD011535
[http://dx.doi.org/10.1002/14651858.CD011535.pub2] [PMID: 2927 1481]

[5] Knezevic I, Griffiths E. WHO standards for biotherapeutics, including biosimilars: An example of the evaluation of complex biological products. Ann N Y Acad Sci 2017; 1407(1): 5-16.

[http://dx.doi.org/10.1111/nyas.13434] [PMID: 28905423]

[6] Li F, Vijayasankaran N, Shen AY, Kiss R, Amanullah A. Cell culture processes for monoclonal antibody production. MAbs 2010; 2(5): 466-79.

[http://dx.doi.org/10.4161/mabs.2.5.12720] [PMID: 20622510]

[7] Mestre-Ferrandiz J, Sussex J, Towse A. The R\&D cost of a new medicine 2012. https://www.ohe.org/publications/rd-cost-new medicine

[8] Modena V, Bianchi G, Roccatello D. Cost-effectiveness of biologic treatment for rheumatoid arthritis in clinical practice: An achievable target? Autoimmun Rev 2013; 12(8): 835-8.

[http://dx.doi.org/10.1016/j.autrev.2012.11.009] [PMID: 23219766]

[9] Blackstone EA, Joseph PF. The economics of biosimilars. Am Health Drug Benefits 2013; 6(8): 469-78.

[PMID: 24991376]

[10] World Health Organization, Expert Committee on Biological Standardization. 2009. http://www.who.int/biologicals/areas/biological therapeutics/BIOTHERAPEUTICS FOR WEB 22APRIL2010.pdf

[11] European Medicines Agency. Guideline on similar biological medicinal products 2014. http://www.ema.europa.eu/docs/en GB/ document_library/Scientific_guideline/2014/10/WC500176768.pdf

[12] US Food and Drug Administration. Considerations in demonstrating interchangeabiity with a reference product 2017 . https://www.fda.gov/ downloads/Drugs/GuidanceComplianceRegulatoryInformation/Guidan ces/UCM537135.pdf

[13] Mysler E, Pineda C, Horiuchi T, et al. Clinical and regulatory perspectives on biosimilar therapies and intended copies of biologics in rheumatology. Rheumatol Int 2016; 36(5): 613-25. [http://dx.doi.org/10.1007/s00296-016-3444-0] [PMID: 26920148]

[14] Sahoo N, Choudhury K, Manchikanti P. Manufacturing of biodrugs: Need for harmonization in regulatory standards. BioDrugs: Clinical immunotherapeutics, biopharmaceuticals and gene therapy 2009; 23 : 217-9.

[http://dx.doi.org/10.2165/11317110-000000000-00000]

[15] McCamish M, Woollett G. Worldwide experience with biosimilar development. MAbs 2011; 3(2): 209-17.

[http://dx.doi.org/10.4161/mabs.3.2.15005] [PMID: 21441787]

[16] Smolen JS, Landewé R, Bijlsma J, et al. EULAR recommendations for the management of rheumatoid arthritis with synthetic and biological disease-modifying antirheumatic drugs: 2016 update. Ann Rheum Dis 2017; 76(6): 960-77.

[http://dx.doi.org/10.1136/annrheumdis-2016-210715] [PMID: 28264 816]

[17] van der Heijde D, Ramiro S, Landewé R, et al. 2016 update of the ASAS-EULAR management recommendations for axial spondyloarthritis. Ann Rheum Dis 2017; 76(6): 978-91.

[http://dx.doi.org/10.1136/annrheumdis-2016-210770] [PMID: 2808 7505]

[18] Al Maini M, Adelowo F, Al Saleh J, et al. The global challenges and opportunities in the practice of rheumatology: White paper by the World Forum on Rheumatic and Musculoskeletal Diseases. Clin Rheumatol 2015; 34(5): 819-29.

[http://dx.doi.org/10.1007/s10067-014-2841-6] [PMID: 25501633]

[19] El Zorkany B, Alwahshi HA, Hammoudeh M, et al. Suboptimal management of rheumatoid arthritis in the Middle East and Africa: could the EULAR recommendations be the start of a solution? Clin Rheumatol 2013; 32(2): 151-9.

[http://dx.doi.org/10.1007/s10067-012-2153-7] [PMID: 23274756]

[20] European Medicines Agency. 2017. http://www.ema.europa.eu/ docs/en_GB/document_library/Leaflet/2017/05/WC500226648.pdf

[21] World Health Organization. WHO Expert Committee on Biological Standardization 2012. http://www.who.int/biologicals/expert_committee/TRS_978_61st_report.pdf

[22] US Food and Drug Administration. Quality considerations in demonstrating biosimilarity of a therapeutic protein product to a reference product 2015. http://www.fda.gov/downloads/Drugs/ Guidance Compliance- Regulatory Information/ Guidances/ UCM29 1134.pdf

[23] US Food and Drug Administration. Scientific considerations in demonstrating biosimilarity to a reference product 2015. http://www. fda.gov/downloads/Drugs/GuidanceComplianceRegulatory Information/Guidances/UCM291128. pdf 
[24] Chisango T. African Union Ministers of Health adopt treaty for the establishment of the African Medicines Agency Treaty to be summited to the Specialised Technical Committee on Justice and Legal Affairs later on this year 2018. https://au.int/en/pressreleases/20180520 /african-union-ministers-health-adopt-treaty-establishment-africanmedicines

[25] Niamane RBR, El Bouchti I, Harzy T, et al. Recommandations de la Société Marocaine de Rhumatologie pour la prise en charge de la polyarthrite rhumatoïde: mise à jour du référentiel de 2011. Rev Mar Rhum 2014; 30: 3-13.

[26] Bahiri RIL, Bezza A, Khalil A, et al. Recommandations de la Société Marocaine de Rhumatologie (SMR) pour la prise en charge thérapeutique des malades atteints de spondylarthrite ankylosante (SpA) grave. Rev Mar Rhum 2017; 39: 3-18

[27] Farhat F, Othman A, El Karak F, Kattan J. Review and results of a survey about biosimilars prescription and challenges in the Middle East and North Africa region. Springerplus 2016; 5(1): 2113. [http://dx.doi.org/10.1186/s40064-016-3779-8] [PMID: 28090427]

[28] Jacobs I, Petersel D, Shane LG, et al. Monoclonal antibody and fusion protein biosimilars across therapeutic areas: A systematic review of published evidence. BioDrugs: Clinical immunotherapeutics, biopharmaceuticals and gene therapy 2016; 30: 489-523.

[http://dx.doi.org/10.1007/s40259-016-0199-9]

[29] Strand V, Balsa A, Al-Saleh J, et al. Immunogenicity of biologics in chronic inflammatory diseases: A systematic review. BioDrug 2017; 2017: 06-15.

[http://dx.doi.org/10.1007/s40259-017-0231-8]

[30] Emery P, Vencovský J, Sylwestrzak A, et al. A phase III randomised, double-blind, parallel-group study comparing SB4 with etanercept reference product in patients with active rheumatoid arthritis despite methotrexate therapy. Ann Rheum Dis 2017; 76(1): 51-7.

[http://dx.doi.org/10.1136/annrheumdis-2015-207588] [PMID: 2615 0601]

[31] Smolen JS, Choe JY, Prodanovic N, et al. Safety, immunogenicity and efficacy after switching from reference infliximab to biosimilar SB2 compared with continuing reference infliximab and SB2 in patients with rheumatoid arthritis: Results of a randomised, double-blind, phase III transition study. Ann Rheum Dis 2018; 77(2): 234-40.

[http://dx.doi.org/10.1136/annrheumdis-2017-211741] [PMID: 2904 2358]

[32] Griffiths CEM, Thaçi D, Gerdes S, et al. The EGALITY study: A confirmatory, randomized, double-blind study comparing the efficacy, safety and immunogenicity of GP2015, a proposed etanercept biosimilar, vs. the originator product in patients with moderate-tosevere chronic plaque-type psoriasis. Br J Dermatol 2017; 176(4): 928-38.

[http://dx.doi.org/10.1111/bjd.15152] [PMID: 27787890]

[33] Moots R CC, Petersel D, Rolland C, Jones H, Mysler E. Efficacy and safety outcomes for originator TNF inhibitors and biosimilars in rheumatoid arthritis and psoriasis trials: A systematic literature review 2018; 32: 193-9.

[http://dx.doi.org/10.1007/s40259-018-0283-4]

[34] Kay J, Isaacs JD. Clinical trials of biosimilars should become more similar. Ann Rheum Dis 2017; 76(1): 4-6.

[http://dx.doi.org/10.1136/annrheumdis-2015-208113] [PMID: 2756 6795]

[35] Moots R, Azevedo V, Coindreau JL, et al. Switching between reference biologics and biosimilars for the treatment of rheumatology, gastroenterology, and dermatology inflammatory conditions: Considerations for the clinician. Curr Rheumatol Rep 2017; 19(6): 37. [http://dx.doi.org/10.1007/s11926-017-0658-4] [PMID: 28623625]

[36] US Food and Drug Administration. Questions and answers on biosimilar development and the BPCI Act 2016. https://www.fda. gov/downloads/drugs/guidances/ucm444661.pdf

[37] Ebbers HC, Muenzberg M, Schellekens H. The safety of switching between therapeutic proteins. Expert Opin Biol Ther 2012; 12(11): $1473-85$.

[http://dx.doi.org/10.1517/14712598.2012.711308] [PMID: 22849511]

[38] Numan S, Faccin F. Non-medical switching from originator tumor necrosis factor inhibitors to their biosimilars: Systematic review of randomized controlled trials and real-world studies. Adv Ther 2018; 35(9): 1295-332.

[http://dx.doi.org/10.1007/s12325-018-0742-9] [PMID: 30084060]

[39] McKinnon RA, Cook M, Liauw W, et al. Biosimilarity and interchangeability: Principles and evidence: A systematic review. BioDrug 2018; 32: 27-52.

[40] Zuñiga L, Calvo B. Biosimilars: Pharmacovigilance and risk management. Pharmacoepidemiol Drug Saf 2010; 19(7): 661-9. [http://dx.doi.org/10.1002/pds.1948] [PMID: 20583204]

[41] Comisión Federal para la Protección contra Riesgos Sanitarios Alerta Sanitaria: La COFEPRIS revoca registro de producto "Kikuzubam" 2014.https://www.gob.mx/cms/uploads/attachment/file/127522/2_Aler ta_sanitaria_KIKUZUBAM_28032014.pdf

[42] Castañeda-Hernández G, González-Ramírez R, Kay J, Scheinberg MA. Biosimilars in rheumatology: What the clinician should know. RMD Open 2015; 1(1)e000010

[http://dx.doi.org/10.1136/rmdopen-2014-000010] [PMID: 26509046]

[43] Isah AO, Pal SN, Olsson S, Dodoo A, Bencheikh RS. Specific features of medicines safety and pharmacovigilance in Africa. Ther Adv Drug Saf 2012; 3(1): 25-34.

[http://dx.doi.org/10.1177/2042098611425695] [PMID: 25083223]

[44] World Health Organization. http://www.who.int/medicines/ services /inn/bq_innproposal201506.pdf.pdf?ua =1

[45] US Food and Drug Administration. Nonproprietary naming of biological products 2017. https://www.fda.gov/downloads/ drugs /guidances/ucm459987.pdf

[46] Rovira J, Espín J, Garcia L, Olry de Labry A. The impact of biosimilars' entry in the EU market 2011. http://citeseerx.ist.psu.edu /viewdoc/download?doi=10.1.1.357.2218\&rep=rep1\&type=pdf

[47] Qunitiles IMS. The impact of biosimilar competition in Europe 2017; Available from: 2017 IMS Biosimilar report - The Impact of Biosimilar Competition in Europe 2017.

[48] 111th U.S. Congress. Patient protection and affordable care act: Public law 111-148 2010. https://www.gpo.gov/fdsys/pkg/PLAW-111 publ148/content-detail.html

[49] Generics and Biosimilars Initiative (GaBi). Biosimilars of adalimumab 2014.

http://www.gabionline.net/Biosimilars/General/Biosimilars-of-adalimu mab

[50] Generics and Biosimilars Initiative (GaBi). Biosimilars of etanercept 2015.

http://www.gabionline.net/Biosimilars/General/Biosimilars-of-etanerc ept

[51] Generics and Biosimilars Initiative (GaBi). Biosimilars of infliximab 2015.

http://www.gabionline.net/Biosimilars/General/Biosimilars-of-inflixim $\mathrm{ab}$

[52] Generics and Biosimilars Initiative (GaBi). Biosimilars of rituximab 2015.

http://www.gabionline.net/Biosimilars/General/Biosimilars-of-rituxim $\mathrm{ab}$

[53] Maity S, Ullanat R, Lahiri S, et al. A non-innovator version of etanercept for treatment of arthritis. Biologicals 2011; 39: 384-95. [http://dx.doi.org/10.1016/j.biologicals.2011.08.014]

[54] Cohen SB, Alonso-Ruiz A, Klimiuk PA, et al. Similar efficacy, safety and immunogenicity of adalimumab biosimilar BI 695501 and Humira reference product in patients with moderately to severely active rheumatoid arthritis: Results from the phase III randomised VOLTAIRE-RA equivalence study. Ann Rheum Dis 2018; 77(6): 914-21.

[http://dx.doi.org/10.1136/annrheumdis-2017-212245] [PMID: 2951 4803]

[55] Genovese MC, Glover J, Matsunaga N, Chisholm DRA. Efficacy, safety and immunogenicity in randomized, double-blind (DB) and open-label extension (OLE) studies comparing FKB327, an adalimumab biosimilar, with the adalimumab reference product (Humira $\left.{ }^{\circledR} ; \mathrm{RP}\right)$ in patients (pts) with active rheumatoid arthritis (RA). Arthritis Rheumatol 2017; 69suppl $10: 2799$.

[56] Weinblatt ME, Baranauskaite A, Dokoupilova E, et al. Switching from reference adalimumab to SB5 (Adalimumab Biosimilar) in patients with rheumatoid arthritis: Fifty-two-week phase III randomized study results. Arthritis Rheumatol 2018; 70(6): 832-40. [http://dx.doi.org/10.1002/art.40444] [PMID: 29439289]

[57] Cohen S, Genovese MC, Choy E, et al. Efficacy and safety of the biosimilar ABP 501 compared with adalimumab in patients with moderate to severe rheumatoid arthritis: A randomised, double-blind, phase III equivalence study. Ann Rheum Dis 2017; 76(10): 1679-87. [http://dx.doi.org/10.1136/annrheumdis-2016-210459] [PMID: 2858 4187]

[58] Cohen S, Pablos JL, Pavelka K, et al. An open-label extension study to demonstrate long-term safety and efficacy of ABP 501 in patients with rheumatoid arthritis. Arthritis Res Ther 2019; 21(1): 84. [http://dx.doi.org/10.1186/s13075-019-1857-3] [PMID: 30922373]

[59] Emery P, Vencovský J, Sylwestrzak A, et al. Long-term efficacy and 
safety in patients with rheumatoid arthritis continuing on SB4 or switching from reference etanercept to SB4. Ann Rheum Dis 2017; 2017annrheumdis-2017-211591

[http://dx.doi.org/10.1136/annrheumdis-2017-211591] [PMID: 2879 4078]

[60] Tanaka Y, Yamanaka H, Takeuchi T, et al. Safety and efficacy of CTP13 in Japanese patients with rheumatoid arthritis in an extension phase or after switching from infliximab. Mod Rheumatol 2017; 27(2): 237-45.

[http://dx.doi.org/10.1080/14397595.2016.1206244] [PMID: 27586 879]

[61] Yoo DH, Prodanovic N, Jaworski J, et al. Efficacy and safety of CTP13 (biosimilar infliximab) in patients with rheumatoid arthritis: Comparison between switching from reference infliximab to CT-P13 and continuing CT-P13 in the PLANETRA extension study. Ann Rheum Dis 2017; 76(2): 355-63.

[http://dx.doi.org/10.1136/annrheumdis-2015-208786] [PMID: 2713 0908]

[62] Taylor P, Wyand M, Knight A, Costantino CCL. Efficacy of the biosimilar BOW015, compared to originator infliximab, initiated at moderate and severe disease activity thresholds in rheumatoid arthritis. Ann Rheum Dis 2016; 75: 488-9.

[http://dx.doi.org/10.1136/annrheumdis-2016-eular.4143]

[63] Park W, Yoo DH, Miranda P, et al. Efficacy and safety of switching from reference infliximab to CT-P13 compared with maintenance of CT-P13 in ankylosing spondylitis: 102-week data from the PLANETAS extension study. Ann Rheum Dis 2017; 76(2): 346-54. [http://dx.doi.org/10.1136/annrheumdis-2015-208783] [PMID: 2711 7698]

[64] Blauvelt A, Lacour JP, Fowler J, et al. Long-term efficacy, safety and immunogenicity results from a randomized, double-blind, Phase III confirmatory efficacy and safety study comparing GP2017, a proposed biosimilar, with reference adalimumab [abstract]. Arthritis Rheumatol 2017; 69(suppl 10): 2240.

[65] Hodge J, Tang H, O'Connor P, Finck B. Switching from adalimumab to CHS-1420: A randomized, double-blind global clinical trial in patients with psoriasis and psoriatic arthritis [abstract]. Arthritis Rheumatol 2017; 69(suppl 10): 2879..

[66] Papp K, Bachelez H, Costanzo A, et al. Clinical similarity of the biosimilar ABP 501 compared with adalimumab after single transition: Long-term results from a randomized controlled, double-blind, 52week, phase III trial in patients with moderate-to-severe plaque psoriasis. Br J Dermatol 2017; 177(6): 1562-74.

[http://dx.doi.org/10.1111/bjd.15857] [PMID: 28755394]

[67] Gerdes S, Thaçi D, Griffiths CEM, et al. Multiple switches between GP2015, an etanercept biosimilar, with originator product do not impact efficacy, safety and immunogenicity in patients with chronic plaque-type psoriasis: 30 -week results from the phase 3, confirmatory EGALITY study. J Eur Acad Dermatol Venereol 2018; 32(3): 420-7. [http://dx.doi.org/10.1111/jdv.14605] [PMID: 28960486]

[68] Jørgensen KK, Olsen IC, Goll GL, et al. Switching from originator infliximab to biosimilar CT-P13 compared with maintained treatment with originator infliximab (NOR-SWITCH): A 52-week, randomised, double-blind, non-inferiority trial. Lancet 2017; 389(10086): 2304-16. [http://dx.doi.org/10.1016/S0140-6736(17)30068-5] [PMID: 28502 609]

[69] Goll GL, Jørgensen KK, Sexton J, et al. Long-term efficacy and safety of biosimilar infliximab (CT-P13) after switching from originator infliximab: Open-label extension of the NOR-SWITCH trial. J Intern Med 2019; 285(6): 653-69.

[http://dx.doi.org/10.1111/joim.12880] [PMID: 30762274]

[70] Volkers A, Jansen J. SIMILAR trial - efficacy of infliximab-biosimilar compared to infliximab biological in patients with inflammatory bowel disease in remission - A randomized, controlled double blind, Phase 4 noninferiority trial. United European Gastroenterol J 2017; 5(Suppl.1): A307.

[71] Ye B, Kim YH, Pesegova M, et al. Phase Iii randomized controlled trial to compare biosimilar infliximab (CT-P13) with innovator infliximab in patients with active Crohn's disease: 1-year maintenance and switching results. Gastroenterology 2018; 154(Suppl.1): S-167-8. [http://dx.doi.org/10.1016/S0016-5085(18)30972-7]

[72] Ye BD, Pesegova M, Alexeeva O, et al. Efficacy and safety of biosimilar CT-P13 compared with originator infliximab in patients with active Crohn's disease: An international, randomised, doubleblind, phase 3 non-inferiority study. Lancet 2019; 393(10182): 1699-707.

[http://dx.doi.org/10.1016/S0140-6736(18)32196-2] [PMID: 30929 895]

\section{2019 Mkinsi et al.}

This is an open access article distributed under the terms of the Creative Commons Attribution 4.0 International Public License (CC-BY 4.0), a copy of which is available at: (https://creativecommons.org/licenses/by/4.0/legalcode). This license permits unrestricted use, distribution, and reproduction in any medium, provided the original author and source are credited. 